\title{
Optical molecular imaging to detect early endothelial activation: a promising novel modality for RA?
}

The ability to detect the inflammatory changes in rheumatoid arthritis (RA) as early as possible is currently a major area of research, as early diagnosis and implementation of treatment can greatly improve the prognosis of patients with this disease. Conventional imaging modalities, such as plain radiography, can only detect RA features that occur late in the disease course, such as bone erosions and joint space narrowing. Molecular imaging modalities, however, have the potential to identify the very early inflammatory events that lead to clinical RA. Gompels and colleagues investigated the capability of in vivo optical molecular imaging using an anti-E-selectin antibody labelled with a near-infrared fluorophore to detect early endothelial activation in mouse models of acute inflammation and RA.

In the mouse model of acute inflammation, tumor necrosis factor was injected into the right foot pad of the animals, leading to localized edema.
Injected anti-E-selectin antibody localized to the inflamed paw, and showed a 2.46-fold increase in fluorescence compared with a control antibody $(P<0.001)$.

In mice with collagen-induced arthritis

$$
\begin{aligned}
& 4 \text {... [molecular imaging] has } \\
& \text { the potential to improve the } \\
& \text { detection of early RA... } 77
\end{aligned}
$$

(CIA), significantly increased fluorescence was observed in arthritic mice versus healthy mice $(P<0.001)$ and in mice injected with anti-E-selectin versus those receiving the control antibody $(P<0.001)$. The main areas of inflammation were shown to be the metatarsal heads and ankle joints. Treatment with etanercept abrogated the arthritis, which correlated with a significant reduction in fluorescence. In animals with no arthritis in one paw (i.e. a clinical score of 0 ) but arthritis in other paws, the nonarthritic paws showed significantly increased fluorescence compared to paws of control, nonimmunized mice $(P<0.01)$, demonstrating the promising sensitivity and specificity of this technique.

The authors conclude that the use of optimal molecular imaging to detect endothelial activation might be an effective method for quantifying disease severity and responses to experimental treatments in animal models. Furthermore, it has the potential to improve the detection of early RA, as endothelial activation is an early feature of the inflammatory process. Indeed, the development of novel reporter probes and fluorescent dyes might lead to further insights into the molecular alterations present early in the disease.

Nick Warde

Original article Gompels, L. L. et al. In vivo fluorescence imaging of E-selectin: quantitative detection of endothelia activation in arthritis. Arthritis Rheum. doi:10.1002/ art.30082 